\title{
On certain upper bounds for the sum of divisors function $\sigma(n)$
}

\author{
József SÁNDOR \\ Babeş-Bolyai University, \\ Faculty of Mathematics and \\ Computer Science, Romania \\ email: jsandor@math.ubbcluj.ro
}

\author{
Lehel István KOVÁCS \\ Sapientia Hungarian University of \\ Transylvania, Department of \\ Mathematics and Informatics, Romania \\ email: klehel@ms.sapientia.ro
}

Abstract. Upper bounds for $\sigma(n)$ are provided in terms of other arithmetic functions as $\varphi(n), d(n), \psi(n), P(n)$, etc. Comparision of older results are given, too.

\section{Introduction}

Let $n>1$ be written in its canonical form

$$
\mathrm{n}=\mathrm{p}_{1}^{\mathrm{a}_{1}} \cdots \mathrm{p}_{\mathrm{r}}^{\mathrm{a}_{\mathrm{r}}}
$$

where $p_{i}$ are distinct primes, $a_{i} \geq 1$ integers, $i=1,2, \ldots, r$.

Then it is well-known the following representations formula for the sum of divisors function $\sigma(n)$, and number of divisors function $d(n)$ :

$$
\sigma(n)=\prod_{i=1}^{r}\left(\frac{p_{i}^{a_{i}+1}-1}{p_{i}-1}\right), d(n)=\prod_{i=1}^{r}\left(a_{i}+1\right)
$$

Similarly, for the Euler's totient $\varphi(n)$, and Dedekind's totient $\psi(n)$ one has:

$$
\varphi(n)=n \prod_{i=1}^{r}\left(1-\frac{1}{p_{i}}\right), \psi(n)=n \prod_{i=1}^{r}\left(1+\frac{1}{p_{i}}\right)
$$

2010 Mathematics Subject Classification: 11A25

Key words and phrases: arithmetic functions, inequalities 
Here $r=\omega(r)$ usually denotes the number of distinct divisors of $n$, in contrast with the total number of prime factors of $n$, which is $a_{1}+a_{2}+\cdots+a_{n}=\Omega(n)$.

In what follows, let $P(n)$ denote the greatest prime factor of $n$. This definition applies for $n>1$, but in (2) and (3) it is obvious the completion for the case $\mathrm{n}=1$, namely:

$$
\sigma(1)=d(1)=\varphi(n)=\psi(1)=1
$$

There are many inequalities for these arithmetical functions; for a survey of results, see the monograph [4], or the recent papers [5], [6], [8], [9], [10], [11].

Partcularly, the following upper bounds for the function $\sigma(n)$ are known:

$$
\begin{aligned}
& \sigma(n) \leq \frac{n^{2}}{\varphi(n)} \text { for } n \geq 1, \\
& \sigma(n) \leq \varphi(n)(d(n))^{2} \text { for } n \geq 2, \\
& \sigma(n) \leq n d(n)-\varphi(n) \text { for } n \geq 2, \\
& \sigma(n) \leq \varphi(n)+d(n)(n-\varphi(n)) \text { for } n \geq 1, \\
& \sigma(n) \leq\left(\frac{n+1}{2}\right) d(n) \text { for } n \geq 1 .
\end{aligned}
$$

We note that inequality (5) has been rediscovered many times in the literature. In a slightly different form it appeared in a paper by O. Meissener from 1907 (see [4], p. 77). Inequality (6) is due to A. Makowski (1974, see [4], p. 11); (7) is due to A. Makowski (1960, see [4], p. 11), while (8) is due to the first author (1989, see [4], p. 10). Finally, (9) is due to E.S. Langford (1978, see [4], p. 86).

An improvement of (6) for odd values of $\mathfrak{n}$, is due to first author (1988, see [4], p. 10):

$$
\sigma(n) \leq \varphi(n) d(n) \text { for } n \geq 1 \text { odd. }
$$

It is easy to see that (10) implies for even values:

$$
\sigma(n)<2 \varphi(n) d(n) \text { for } n \geq 2 \text { even. }
$$

In the same year, K.T. Atanassov (see [4], p. 88) proved the upper bounds:

$$
\begin{aligned}
& \sigma(n) \leq \varphi(n) P(n) \text { for } n \text { odd, } \\
& \sigma(n)<4 \varphi(n) P(n) \text { for } n \text { even. }
\end{aligned}
$$

Here, as above, relation (13) is an immediate consequence of (12). 
Remark 1 As $\mathrm{d}(\mathrm{n})$ and $\mathrm{P}(\mathrm{n})$ are not generally comparable, inequalities (10) and (11) are independent of each other. For any $\mathrm{n}=\mathrm{p}=$ prime, one has $\mathrm{d}(\mathrm{n})=2<\mathrm{P}(\mathrm{n})$, so (10) is better than (12). Also for $\mathrm{n}=\mathrm{p}^{2}$, when $\mathrm{d}(\mathrm{n})=$ $3 \leq \mathrm{P}(\mathrm{n})$ for $\mathrm{n}$ odd. However, even for prime powers $\mathrm{n}=\mathrm{p}^{\mathrm{a}}$, when $\mathrm{p}>\mathrm{a}+1$, clearly (12) will be stronger than (10).

\section{Main results}

\section{New inequalities}

One of the aims of this paper is to offer an improvement of (12) and (13); as follows:

Theorem 1 One has

$$
\sigma(n)<\frac{3}{4} \varphi(n) P(n) \text { for } n \geq 3 \text { odd }
$$

and

$$
\sigma(n)<3 \varphi(n) P(n) \text { for } n \text { even. }
$$

Proof. The following auxiliary result by R. A. Rankin (1963, see [1], p. 193) will be used:

Lemma 1 For all $\mathrm{n} \geq 1$ one has

$$
\frac{1 \cdot 3 \cdot 5 \cdots(2 n-1))}{2 \cdot 4 \cdot 6 \cdots 2 n} \leq \sqrt{\frac{3 / 4}{2 n+1}}
$$

Now, as a consequence, we can deduce an upper bound for $\left(\frac{n}{\varphi(n)}\right)^{2}=$ $\prod_{p \mid n}\left(\frac{p}{p-1}\right)^{2} \leq \frac{3^{2}}{2^{2}} \cdot \frac{5^{2}}{4^{2}} \cdots \frac{(2 m+1)^{2}}{(2 m)^{2}}$, where we have denoted the greatest prime divisior of $n$ as $2 m+1$. Now, remark that by (16) one has $\frac{3 \cdot 5 \cdots(2 m+1)}{2 \cdot 4 \cdots(2 m)} \leq$ $\sqrt{\frac{3}{4}(2 m+1)}=\sqrt{\frac{3}{4} \mathrm{P}(\mathrm{n})}$, which implies relation (14), by remarking that by (5) one has $\sigma(n)<\left(\frac{n}{\varphi(n)}\right)^{2} \cdot \varphi(n) \leq \frac{3}{4} \varphi(n) P(n)$, for $n \geq 3$ odd, since in (5) there is equality only for $n=1$. If $n=2^{k} N(k \geq 1, N$ odd $)$ is an even integer, then $P(n)=P(N), \varphi(n)=2^{k-1} \varphi(N)$ and $\sigma(n)=\left(2^{k+1}-1\right) \sigma(N)$, so (15) follows from (13) by $2^{k+1}-1<2^{k+1}$ and $4 \frac{3}{4}=3$. 
Remark 2 From the proof of Theorem 1 we can remark that

$$
\left(\frac{\mathrm{n}}{\varphi(\mathrm{n})}\right)^{2} \leq \frac{3}{4} \mathrm{P}(\mathrm{n}) \text { for } \mathrm{n} \geq 3 \text { odd }
$$

and

$$
\left(\frac{n}{\varphi(n)}\right)^{2} \leq 3 P(n) \text { for } n \text { even, }
$$

which improve the classical inequality (see [5])

$$
\frac{\mathrm{n}}{\varphi(\mathrm{n})} \leq \mathrm{P}(\mathrm{n}) \text { for all } \mathrm{n} \geq 2
$$

This follows by $\left(\frac{\mathrm{n}}{\varphi(\mathrm{n})}\right)^{2} \geq \frac{\mathrm{n}}{\varphi(\mathrm{n})}$. Clearly, (17) improves (19) for all odd $\mathrm{n}$, while (18) improves (19) for all $\mathrm{n} \neq 2^{\mathrm{k}}$ (i.e. powers of 2). Indeed, $3 \mathrm{P}(\mathrm{n}) \leq$ $P^{2}(n)$ only if $\mathrm{P}(\mathrm{n}) \geq 3$, and for even $\mathrm{n}$ this is true for $\mathrm{n} \neq 2^{\mathrm{k}}$.

Theorem 2 One has

$$
\sigma(\mathrm{n})<\psi(\mathrm{n})+\sigma(\mathrm{n}) \cdot \frac{3}{8} \mathrm{P}(\mathrm{n}) \text { for } \mathrm{n} \text { odd, }
$$

and

$$
\sigma(n)<\psi(n)+\sigma(n) \cdot \frac{3}{2} P(n) \text { for } n \text { even }
$$

\section{Proof.}

The proof of the following auxiliary result may be found in [5]:

Lemma 2 For all $\mathrm{n} \geq 1$ one has

$$
2 \cdot \frac{\psi(n)}{n} \geq 1+\frac{n}{\varphi(n)}
$$

Now, by (5) and (22) one can write: $\sigma(n)-\psi(n)<\frac{n^{2}}{\varphi(n)}-\frac{n}{2}-\frac{n^{2}}{2 \varphi(n)}$, so

$$
\frac{\sigma(n)-\psi(n)}{\varphi(n)}<\frac{n}{2 \varphi(n)} \cdot\left(\frac{n}{\varphi(n)}-1\right) \text { for } n>1 .
$$

Now, (20) and (21) are consequences of (17) and (18) as applications to (23). 
Theorem 3 One has

$$
\sigma(n)<\psi(n)+\frac{k \cdot n^{2}}{\varphi(n)}
$$

where $\mathrm{k}=1-\frac{6}{\pi^{2}}=0.392 \ldots$

For all odd $\mathrm{n} \geq 3$ one has

$$
\sigma(\mathrm{n})<\psi(\mathrm{n})+\frac{3}{10} \varphi(\mathrm{n}) \mathrm{P}(\mathrm{n}) .
$$

Proof. We shall use the following inequality from [6]:

$$
\psi(n)>\frac{c \cdot n^{2}}{\varphi(n)}, n \geq 1
$$

where $c=6 / \pi^{2}$. Now, by (5) and (26) one has $\sigma(n)-\psi(n)<\frac{n^{2}}{\varphi(n)}-\frac{c^{2}}{\varphi(n)}=$ $(1-c) \frac{n^{2}}{\varphi(n)}=\frac{k n^{2}}{\varphi(n)}$, which proves relation (24). Now, as $\frac{n^{2}}{\varphi(n)}=\varphi(n) \cdot\left(\frac{n}{\varphi(n)}\right)^{2}$, and by (17) we get (18), by remarking that $\frac{3}{4} k=0.294 \cdots<0.3=\frac{3}{10}$.

Remark 3 Relation (25) improves slightly (20), as $\frac{3}{10}<\frac{3}{8}$.

Theorem 4 One has

$$
\sigma(n)<\frac{\pi^{2}}{6} \cdot \psi(n), n \geq 1
$$

For all odd $\mathrm{n}$ one has

$$
\sigma(n)<\psi(n)+a \cdot \varphi(n) \cdot 2^{\omega(n)},
$$

where $\mathrm{a}=\pi^{2} / 6-1$

Proof. For inequality (27) see paper [6]. For (28) use (27) and the remark that $\frac{\psi(n)}{\varphi(n)}=\prod_{p \mid n} \frac{p+1}{p-1} \leq 2^{\omega(n)}$ since $\frac{p+1}{p-1} \leq 2$ for $p \geq 3$ (i.e. $n=$ odd). Therefore, we can write $\sigma(n)-\psi(n)<a \cdot \psi(n)=a \cdot \varphi(n) \cdot\left(\frac{\psi(n)}{\varphi(n)}\right)<a \varphi(n) \cdot 2^{\omega(n)}$.

Remark 4 As $0<\mathrm{a}<1$, from (28) we get also

$$
\sigma(\mathrm{n})<\psi(\mathrm{n})+\varphi(\mathrm{n}) \cdot 2^{\omega(\mathrm{n})}, \mathrm{n} \text { odd. }
$$


When $\mathrm{n}$ is squarefull, this improves the following inequality by K.T. Atanassov (see [12]):

$$
\sigma(n)<\psi(n)+\varphi(n) \cdot 2^{\Omega(n)-\omega(n)}, n \geq 1 .
$$

Indeed, if $n$ is squarefull (i.e., when in (1) all $a_{i} \geq 2$ for $i=1,2, \ldots, r$ ), we get $\Omega(n)=a_{1}+\cdots+a_{r} \geq 2 r=2 \omega(n)$, so $\omega(n) \leq \Omega(n)-\omega(n)$, and (29) refines (30).

\section{Comparison of upper bounds for $\sigma(n)$}

Many times, there have been published various inequalities containing also other arithmetic functions, but without comparison to each other. For example, it is not remarked in the literature that, inequality (5) is stronger than (6):

Theorem 5 For all $\mathrm{n} \geq 1$

$$
\sigma(n) \leq \frac{n^{2}}{\varphi(n)} \leq \varphi(n)(d(n))^{2},
$$

i.e., inequality (5) implies inequality (6).

Proof. The second inequality of (31) may be rewritten as

$$
\varphi(n) d(n) \geq n,
$$

which is a known inequality of R. Sivaramakrishnan (1967, see [4], p. 10). The following improvement of (32) is due to the first author (1989, see [4], p. 10):

$$
\varphi(n) d(n) \geq \varphi(n)+n-1, n \geq 1 .
$$

Inequality (10) improves also (6) for odd values of $n$. The following result improves (10):

\section{Theorem 6}

$$
\sigma(n) \leq \frac{\psi(n) \cdot d(n)}{2^{\omega(n)}}, n \geq 1 .
$$

For odd $\mathrm{n}$, one has

$$
\sigma(n) \leq \frac{\psi(n) \cdot d(n)}{2^{\omega(n)}} \leq \varphi(n) d(n) .
$$


Proof. Inequality (34) is due to the first author (1988, see [2]). Now, the socond inequality of $(35)$ can be written as $\frac{\psi(\mathfrak{n})}{\varphi(n)} \leq 2^{\omega(\mathfrak{n})}, \mathrm{n}$ odd, which is proved earlier (see the proof of Theorem 4).

Theorem 7 For all $\mathrm{n} \geq 1$,

$$
\sigma(n) \leq n \cdot[\omega(n)+1] .
$$

For $\mathrm{n} \neq$ prime one has

$$
\sigma(n) \leq n \cdot[\omega(n)+1] \leq n \cdot \Omega(n) .
$$

Proof. Inequality (36) appears in the first author's paper [3] from 1989, and it improves the better-known inequality due to R. L. Duncan (1967, see [4], p. 79):

$$
\sigma(n)<n \cdot\left[\frac{7 \omega(n)+10}{6}\right], n \geq 1
$$

Indeed, it is easy to see that, $\omega(\mathfrak{n})+1<\frac{7 \omega(\mathfrak{n})+10}{6}$.

We shall offer here a simple proof of (36). Assume that in the prime factorization (1) one has $p_{1}<\cdots<p_{r}$. Then $p_{1} \geq 2, p_{2} \geq 3, \cdots, p_{r} \geq r+1$, so we get by $(3) \varphi(n) \geq n \cdot\left(1-\frac{1}{2}\right) \cdots\left(1-\frac{1}{r+1}\right)=n \cdot \frac{1}{2} \cdot \frac{2}{3} \cdots \frac{r}{r+1}=\frac{n}{r+1}$, giving:

$$
\varphi(n) \geq \frac{n}{\omega(n)+1}, n \geq 1 .
$$

Now, inequality (36) is a consequence of (5) combined with (39). The second inequality of (37) is true, if $\Omega(n)-\omega(n) \geq 1$. This holds only if in the prime factorization (1) one has that $\Omega(n) \neq \omega(n)$, i.e. if $n \neq$ squarefree (i.e. $\left.\mathrm{n}=\mathrm{p}_{1} \cdots \mathrm{p}_{\mathrm{r}}\right)$. The inequality

$$
\sigma(n) \leq n \cdot \Omega(n), n \neq \text { prime, }
$$

is due to first author (1988, see [4], p. 87). In fact, a new proof of (40) will be offered here, if we prove that, it is true for any $n=p_{1} \cdot p_{2} \cdots p_{r}\left(p_{i}\right.$ distinct primes), for $r \geq 2$. Equivalently,

$$
\left(p_{1}+1\right) \cdots\left(p_{r}+1\right) \leq p_{1} \cdots p_{r} \cdot r, r \geq 2 .
$$

As $\left(1+\frac{1}{p_{1}}\right) \cdots\left(1+\frac{1}{p_{r}}\right) \leq\left(1+\frac{1}{1}\right)\left(1+\frac{1}{3}\right) \cdots\left(1+\frac{1}{r}\right)=\frac{3}{2} \cdot \frac{4}{3} \cdots \frac{r+1}{r}=\frac{r+2}{2} \leq r$ for $r \geq 2$, inequality (40) is proved. 
Remark 5 The above proof shows that in fact

$$
\frac{\psi(n)}{n} \leq \frac{\omega(n)+2}{2}, n \geq 2
$$

Theorem 8 If $\omega(\mathrm{n}) \geq 3$, then

$$
\sigma(n)<n[\omega(n)+1]<d(n) \cdot\left(\frac{n+1}{2}\right),
$$

(i.e. (36) is stronger than (9)). If $\omega(\mathrm{n})=1$ and $\mathrm{n}=\mathrm{p}^{\mathrm{a}}$ (p prime, $\mathrm{a} \geq 1$ ), then for $\mathrm{a} \geq 3$, (43) is true. If $\omega(\mathrm{n})=2$ and $\mathrm{n}$ not squarefree, then (43) is again true. If $\mathrm{\omega}(\mathrm{n})=1$ and $\mathrm{n}=\mathrm{p}^{\mathrm{a}}$ with $\mathrm{a} \in\{1,2\}$ or $\mathrm{n}=$ squarefree, one has

$$
\sigma(n) \leq d(n) \cdot\left(\frac{n+1}{2}\right)<n[\omega(n)+1] .
$$

Proof. As $d(n) \geq 2^{\omega(n)}$, it is sufficient to prove that

$$
2^{\omega(n)-1} \cdot(n+1) \geq n[\omega(n)+1] .
$$

The inequality

$$
2^{k-1} \geq k+1, k \geq 3
$$

can be proved immediately by induction. By letting $k=\omega(n)$, since $n+1>n$, the proof of (41) is complete. Clearly, all inequalities are strict.

If $n=p^{a}$ ( $p$ prime), then $d(n)=a+1 \geq 4$, for $a \geq 3$ and $d(n) \geq 4>\frac{4 n}{n+1}$, so again (41) is true with strict inequality.

If $n \neq p q$ then $n=p^{a} \cdot q^{b}$, where at least one of $a$ and $b \geq 2$. In this case $d(n)=(a+1)(b+1) \geq 2 \cdot 3=6$. On the other hand, one has $6>\frac{6 n}{n+1}$, so again get the strict inequality.

For $n=p$ one has $2 \cdot\left(\frac{p+1}{2}\right)<p \cdot 2$, while for $n=p^{2}, 3 \cdot\left(\frac{p^{2}+1}{2}\right)<p^{2} \cdot 2$ by $3<p^{2}(p \geq 2)$.

Finally, for $n=p q$, we have $4 \cdot\left(\frac{p q+1}{2}\right)<3 p q$ by $p q>2$.

Remark 6 Therefore (43) is true for all $\mathrm{n}>1$ which are not primes, or square of primes, or which are not the product of two distinct primes.

As a comparison of (8) and (9), the following holds true: 
Theorem 9 For all $\mathrm{n}>2$ even numbers one has

$$
\sigma(n)<d(n) \cdot\left(\frac{n+1}{2}\right)<\varphi(n)+d(n)(n-\varphi(n)) .
$$

When $\mathrm{n}$ is odd, generally (8) and (9) are not comparable.

Proof. The first inequality of (47) is strict as in (9) there is equality only for $\mathrm{n}=$ prime. The second inequality may be written also as

$$
d(n) \cdot\left(\frac{2 \varphi(n)-n+1}{2}\right)<\varphi(n) .
$$

Now, if $\boldsymbol{n}$ is even number, it is well-known (and it easily follows by the first relation of (2)) that $\varphi(n) \leq \frac{n}{2}$. This implies that

$$
2 \varphi(n)-n+1 \leq 1 .
$$

Now, if eventually $2 \varphi(n)-n+1 \leq 0$, then (48) is trivially true. Otherwise, we will use besides (47), the following known inequality (see [4], p. 11):

$$
d(n)<\varphi(n) \text { for } n>30 .
$$

Then inequality (48) holds true for all even $n>30$. A particular verification shows that, in fact (48) holds true for all even numbers $4 \leq n \leq 30$. This proves the first part of the theorem.

Let $n=p^{2}$, where $p \geq 5$ is prime. As $d\left(p^{2}\right)=3$ and $\varphi\left(p^{2}\right)=p^{2}-p$, it is immediate that the second inequality of $(47)$ holds in reverse order.

The same can be verified for $n=5 p$, where $p \geq 7$ is a prime.

On the other hand, for $n=3 p$ ( $p \geq 5$ prime), the inequality holds in this order. Therefore, there are infinitely many odd values of $n$ for which the inequality is true in both senses.

Remark 7 Since (8) may be written also as

$$
\sigma(n)+\varphi(n) \leq n d(n)+\varphi(n)(2-d(n)),
$$

by $2-\mathrm{d}(\mathrm{n}) \leq 0$, clearly this inequality strongly refines relation (7). Another refinement of (7), namely

$$
\sigma(n)+\varphi(n) \leq n \cdot 2^{\omega(n)},
$$

is due to C.A. Nicol (1996, see [4], p. 10).

When $\mathrm{n}$ is squarefree (i.e., a product of distinct primes), then, as $\mathrm{nd}(\mathrm{n})=$ $2^{\omega(\mathrm{n})}$ and $2 \leq \mathrm{d}(\mathrm{n})$, clearly (51) is stronger than (52).

It is easy to verify that for $\mathrm{n}=2^{\mathrm{k}}(\mathrm{k} \geq 1)$, (52) is stronger than (51). 
An inequality refining (9) for all $n$, will be provided by

Theorem 10 One has

$$
\sigma(n) \leq \frac{d(n) \sigma^{*}(n)}{2^{w(n)}} \leq d(n) \cdot\left(\frac{n+1}{2}\right), n \geq 1,
$$

where $\sigma^{*}(n)=\prod_{i=1}^{r}\left(p_{i}^{a_{i}}+1\right)$ (for the prime factorization (1) of $\left.n>1\right), \sigma^{*}(1)=$ 1 , denotes the sum of unitary divisors of $n$ (see [4]).

Proof. The first inequality of (53) is published in first author's paper [7] (1994), as an application of an inequality of Klamkin (see also [8])

For the second relation of (53), apply the following auxiliary result:

Lemma 3 For $x_{i} \geq 1$ real numbers $(i=1,2, \ldots, r)$ we have

$$
\prod_{i=1}^{r}\left(x_{i}+1\right) \leq 2^{r-1} \cdot\left(\prod_{i=1}^{r} x_{i}+1\right) \text {. }
$$

This result is well-known, see e.g. [1].

Apply now (54) for $x_{i}=p_{i}^{a_{i}}, r=\omega(n)$, where $n$ has prime factorization (1). Then we get

$$
\sigma^{*}(n) \leq 2^{\omega(n)-1} \cdot(n+1), n>1
$$

which gives the second inequality of (53).

Remark 8 As $\psi(n)=\prod_{i=1}^{r}\left(p_{i}^{a_{i}}+p_{i}^{a_{i}-1}\right)$, clearly $\sigma^{*}(n) \leq \psi(n)$, so the first inequality of (53) offers a refinement of inequality (34).

It is a natural question if (34) and (53) may be further compared. The following result answers this question:

Theorem 11 For $\omega(\mathrm{n}) \geq 2$ one has

$$
\frac{\psi(n) d(n)}{2^{w(n)}}<d(n) \cdot \frac{n+1}{2} .
$$


Proof. By inequality (42) it will be sufficient to study

$$
n \cdot \frac{\omega(n)+2}{2} \leq 2^{\omega(n)} \cdot \frac{n+1}{2} .
$$

For $\omega(n)=1$ this becomes $3 n \leq 2(n+1)$, which is false.

Assume $\omega(n) \geq 2$. Then, as $2^{k} \geq k+2$ for any $k \geq 2$, by letting $k=\omega(n)$, and remarking that $n+1>n,(57)$ immediately follows.

Remark 9 Therefore, one has the following completion to Theorem 10:

$$
\sigma(n) \leq \frac{d(n) \cdot \sigma^{*}(n)}{2^{\omega(n)}} \leq \frac{d(n) \cdot \psi(n)}{2^{\omega(n)}}<d(n) \cdot \frac{n+1}{2}, \text { for } \omega(n) \geq 2 .
$$

Remark 10 In 2010 the first author (see [9]) proved a refinement of a new type of inequality (7):

$$
\sigma(n) \leq \frac{n d(n)-\varphi(n)}{\omega(n)}, \text { for } n \geq 2 \text { and distinct from } 6
$$

which clearly gives another improvement of (7), related to (58):

$$
\sigma(n) \leq \frac{n d(n)-\varphi(n)}{2}<\frac{n d(n)}{2} \text {, for } \omega(n) \geq 2 \text { and } n \text { distinct from } 6
$$

For inequalities related to the weaker relation of (60), see also [5].

Theorem 12 1) The following improvement of (9) holds true:

$$
\sigma(n)<\frac{n d(n)-\varphi(n)}{2}<\frac{n-1}{2} \cdot d(n) \text { for } \omega(n) \geq 2 \text { and } n>30 \text {; }
$$

2) The inequality

$$
\sigma(n) \leq \frac{n-1}{2} \cdot d(n)
$$

holds true for i) $\omega(\mathrm{n}) \geq 2$ and $\mathrm{n}$ distinct from 6 . There is equality only for $\mathrm{n}=10$. ii) if $\mathrm{\omega}(\mathrm{n})=1$, let $\mathrm{n}=\mathrm{p}^{\mathrm{k}}$ (p prime, $\left.\mathrm{k} \geq 1\right)$. Then (62) is true if: a) $\mathrm{k}=2$ and $\mathrm{p} \geq 5$; b) $\mathrm{k} \geq 3$ and $\mathrm{p} \geq 5$; c) $\mathrm{k} \geq 3$ and $\mathrm{p}=3$; d) $\mathrm{k} \geq 4$ and $p=2$. 
Proof. 1) Applying inequality (59) for $\omega(n) \geq 2$ and $n$ distinct from 6 , the first inequality of (61) follows. The inequality is strict, since in [9] it is proved that in (59) there is equality only for $n=10$. The second inequality follows by remarking that one can apply relation (50) for $n>30$.

2) i) By (61), relation (62) holds true for $\omega(n) \geq 2$ and $n>30$. A simple verification for $n=10,12,14,15,18,20,21,22,24,26,28$, which are the $n$ with $\omega(n)=2$, and $n<30$ shows that (62) is not true only for $n=6$. Also, it is true for $n=10$, with equality.

ii) For $n=p^{k}$ inequality (62) becomes

$$
\frac{p^{k+1}-1}{p-1}<\frac{p^{k}-1}{2} \cdot(k+1) .
$$

This inequality is not true for $k=1$ (i.e. $n=p=$ prime). Let $k=2$. Then (63) becomes after a simple transformation: $p(p-2)>5$. This is clearly true only for $p \geq 5$, so case a) is proved.

Apply now the Cauchy mean-value theorem of differential calculus to the functions $f(x)=x^{k+1}$ and $g(x)=x^{k}$ on the interval $[1, p]$, by obtaining: $\frac{f(p)-f(1)}{g(p)-g(1)}=\frac{f(c)}{g(c)}$, where $c$ is in $(1, p)$. We get in this particular case: $\frac{p^{k+1}-1}{p^{k}-1}=$ $\frac{(k+1) \cdot c^{k}}{k \cdot c^{k-1}}=\frac{k+1}{k} \cdot c<(k+1) \cdot \frac{p}{k}$.

Now, we have that $\frac{p}{k}<\frac{p-1}{2}$ for all $k \geq 3$ and $p \geq 5$, so case b) follows.

For $k \geq 3$ clearly we have to consider only the remaining cases $p=2$ and $p=3$. For $p=2$ we have $n=2^{k}$, and the inequality can be written equivalently as $2^{k} \cdot(k-3)>k$. This is true only for $k \geq 4$ (mathematical induction). Let now $p=3$. Then we get the inequality $3^{k} \cdot(k-2)>k$, and this holds only for $k \geq 3$. Therefore, cases c) and d) are completely proved.

\section{References}

[1] D. S. Mitrinović, Analytic inequalities, Springer Verlag, 1970.

[2] J. Sándor, On Dedekind's arithmetical function, Seminarul de t. structurilor, Univ. Timişoara, 51 (1988), 1-15.

[3] J. Sándor, Some diophantine equations for particular arithmetic functions (Romanian), Seminarul de t. structurilor, Univ. Timişoara, 53 (1989), $1-10$.

[4] J. Sándor, D. S. Mitrinović, B. Crstici, Handbook of number theory I., Springer Verlag, 2006 (first ed. by Kluwer Acad. Publ., 1995). 
[5] J. Sándor, On an arithmetic inequality, Anal. Şt. Univ. Ovidius Constanţa, 22 (1) (2014), 257-261.

[6] J. Sándor, On certain inequalities for $\sigma, \varphi, \psi$ and related functions, Notes Number Th. Discr. Math., 20 (2) (2014), 52-60.

[7] J. Sándor, On an inequality of Klamkin with arithmetical applications, Int. J. Math. Ed. Sci. Techn., 25 (1994), 157-158.

[8] J. Sándor, On an inequality of Klamkin, Proc. Jangjeon Math. Soc., 13 (2010), 49-54.

[9] J. Sándor, Two arithmetic inequalities, Adv. Stud. Contemp. Math., 20 (2) (2010), 197-202.

[10] J. Sándor, L. Kovács, A note on the arithmetical functions d(n) and $\sigma(n)$, Octogon Math. Mag., 16 (1A) (2008), 270-274.

[11] J. Sándor, L. Kovács, On an arithmetic inequality by K. T. Atanassov, Proc. Jangjeon Math. Soc., 13 (3) (2010), 313-319.

[12] K. T. Atanassov, Note on $\varphi, \psi$ and $\sigma$-functions. Part 7, Notes Number Th. Discr. Math., 20 (3) (2014), 50-53. 